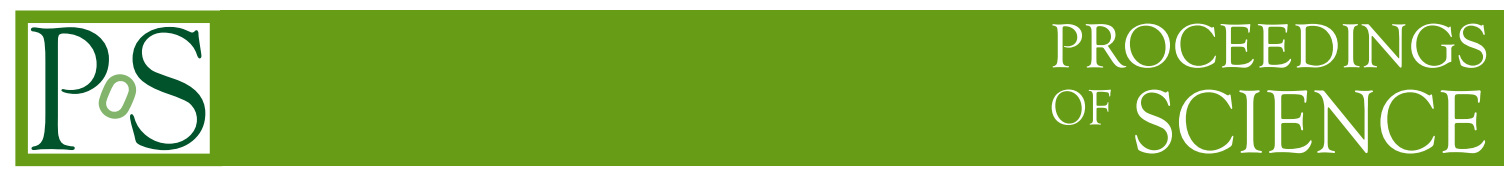

\title{
$100 \mathrm{TeV}$ Gamma-Ray Observation of the Crab Nebula with the Tibet Air Shower Array
}

M. Amenomori, ${ }^{1}$ Y.-W. Bao, ${ }^{2}$ X. J. Bi, ${ }^{3}$ D. Chen, ${ }^{4}$ T. L. Chen, ${ }^{5}$ W. Y. Chen, ${ }^{3}$ Xu Chen, ${ }^{3}$ Y. Chen, ${ }^{2}$ Cirennima, ${ }^{5}$ S. W. Cui, ${ }^{7}$ Danzengluobu, ${ }^{5}$ L. K. Ding, ${ }^{3}$ J. H. Fang, ${ }^{3,6}$ K. Fang, ${ }^{3}$ C. F. Feng, ${ }^{8}$ Zhaoyang Feng, ${ }^{3}$ Z. Y. Feng, ${ }^{9}$ Qi Gao, ${ }^{5}$ Q. B. Gou, ${ }^{3}$ Y. Y. Guo, ${ }^{3}$ Y. Q. Guo, ${ }^{3}$ H. H. He,${ }^{3}$ Z. T. He, ${ }^{7}$ K. Hibino, ${ }^{10}$ N. Hotta, ${ }^{11}$ Haibing Hu, ${ }^{5}$ H. B. Hu, ${ }^{3}$ J. Huang, ${ }^{3}$ H. Y. Jia, ${ }^{9}$ L.Jiang, ${ }^{3}$ H.-B. Jin, ${ }^{4}$ F. Kajino, ${ }^{12}$ K. Kasahara, ${ }^{13}$ Y. Katayose,${ }^{14}$

C. Kato, ${ }^{15}$ S. Kato, ${ }^{16}$ K. Kawata ${ }^{16}{ }^{16}$ W. Kihara, ${ }^{15}$ Y. Ko, ${ }^{15}$ M. Kozai, ${ }^{17}$ Labaciren, ${ }^{5}$ G. M. Le, ${ }^{18}$ A. F. Li, ${ }^{19,8,3}$ H. J. Li, ${ }^{5}$ W. J. Li,,${ }^{3,9}$ Y.-H. Lin, ${ }^{3,6}$ B. Liu, ${ }^{2}$ C. Liu, ${ }^{3}$ J. S. Liu, ${ }^{3}$ M. Y. Liu, ${ }^{5}$ W. Liu, ${ }^{3}$ Y.-Q. Lou, ${ }^{20}$ H.Lu, ${ }^{3}$ X. R. Meng, ${ }^{5}$ H. Mitsui, ${ }^{14}$ K. Munakata, ${ }^{15}$ H. Nakada, ${ }^{14}$ Y. Nakamura, ${ }^{3}$ H. Nanjo, ${ }^{1}$ M. Nishizawa, ${ }^{21}$ M. Ohnishi, ${ }^{16}$ T. Ohura,,${ }^{14}$ S. Ozawa, ${ }^{22}$ X. L. Qian, ${ }^{23}$ X. B. Qu, ${ }^{24}$ T. Saito, ${ }^{25}$ M. Sakata, ${ }^{12}$ T. K. Sako, ${ }^{16}$ Y. Sengoku, ${ }^{14}$ J. Shao, ${ }^{3,8}$ M. Shibata, ${ }^{14}$ A. Shiomi, ${ }^{26}$ H. Sugimoto, ${ }^{27}$ W. Takano, ${ }^{10}$ M. Takita, ${ }^{16}$ Y. H. Tan, ${ }^{3}$ N. Tateyama, ${ }^{10}$ S. Torii, ${ }^{28}$ H. Tsuchiya, ${ }^{29}$ S. Udo, ${ }^{10}$ H. Wang,,${ }^{3}$ H. R. Wu, ${ }^{3}$ L. Xue,${ }^{8}$ K. Yagisawa, ${ }^{14}$ Y. Yamamoto, ${ }^{12}$ Z. Yang, ${ }^{3}$ Y. Yokoe,,${ }^{16}$ A. F. Yuan, ${ }^{5}$

L. M. Zhai, ${ }^{4}$ H. M. Zhang, ${ }^{3}$ J. L. Zhang, ${ }^{3}$ X. Zhang, ${ }^{2}$ X. Y. Zhang, ${ }^{8}$ Y. Zhang, ${ }^{3}$ Yi Zhang, ${ }^{3}$ Ying Zhang, ${ }^{3}$ S. P. Zhao ${ }^{3}$ Zhaxisangzhu, ${ }^{5}$ and X. X. Zhou ${ }^{9}$ (The Tibet AS $\gamma$ Collaboration) 


\author{
${ }^{1}$ Department of Physics, Hirosaki University, Hirosaki 036-8561, Japan \\ ${ }^{2}$ School of Astronomy and Space Science, Nanjing University, Nanjing 210093, China \\ ${ }^{3}$ Key Laboratory of Particle Astrophysics, Institute of High Energy Physics, Chinese Academy of Sciences, \\ Beijing 100049, China \\ ${ }^{4}$ National Astronomical Observatories, Chinese Academy of Sciences, Beijing 100012, China \\ ${ }^{5}$ Physics Department of Science School, Tibet University, Lhasa 850000, China \\ ${ }^{6}$ University of Chinese Academy of Sciences, Beijing 100049, China \\ ${ }^{7}$ Department of Physics, Hebei Normal University, Shijiazhuang 050016, China \\ ${ }^{8}$ Department of Physics, Shandong University, Jinan 250100, China \\ ${ }^{9}$ Institute of Modern Physics, SouthWest Jiaotong University, Chengdu 610031, China \\ ${ }^{10}$ Faculty of Engineering, Kanagawa University, Yokohama 221-8686, Japan \\ ${ }^{11}$ Utsunomiya University, Utsunomiya 321-8505, Japan \\ ${ }^{12}$ Department of Physics, Konan University, Kobe 658-8501, Japan \\ ${ }^{13}$ Shibaura Institute of Technology, Saitama 337-8570, Japan \\ ${ }^{14}$ Faculty of Engineering, Yokohama National University, Yokohama 240-8501, Japan \\ ${ }^{15}$ Department of Physics, Shinshu University, Matsumoto 390-8621, Japan \\ ${ }^{16}$ Institute for Cosmic Ray Research, University of Tokyo, Kashiwa 277-8582, Japan \\ ${ }^{17}$ Institute of Space and Astronautical Science, Japan Aerospace Exploration Agency (ISAS/JAXA), \\ Sagamihara 252-5210, Japan \\ ${ }^{18}$ National Center for Space Weather, China Meteorological Administration, Beijing 100081, China \\ ${ }^{19}$ School of Information Science and Engineering, Shandong Agriculture University, Taian 271018, China \\ ${ }^{20}$ Physics Department, Astronomy Department and Tsinghua Center for Astrophysics, Tsinghua-National \\ Astronomical Observatories of China joint Research Center for Astrophysics, Tsinghua University, Beijing \\ 100084, China \\ ${ }^{21}$ National Institute of Informatics, Tokyo 101-8430, Japan \\ ${ }^{22}$ Advanced ICT Research Institute, National Institute of Information and Communication Technology, \\ Koganei 184-8795, Japan \\ ${ }^{23}$ Department of Mechanical and Electrical Engineering, Shandong Management University, Jinan \\ 250357, China \\ ${ }^{24}$ College of Science, China University of Petroleum, Qingdao, 266555, China \\ ${ }^{25}$ Tokyo Metropolitan College of Industrial Technology, Tokyo 116-8523, Japan \\ ${ }^{26}$ College of Industrial Technology, Nihon University, Narashino 275-8576, Japan \\ ${ }^{27}$ Shonan Institute of Technology, Fujisawa 251-8511, Japan \\ ${ }^{28}$ Research Institute for Science and Engineering, Waseda University, Tokyo 169-8555, Japan \\ ${ }^{29}$ Japan Atomic Energy Agency, Tokai-mura 319-1195, Japan
}

\title{
E-mail: kawatadicrr.u-tokyo.ac.jp
}

The Tibet air shower (AS) array and underground water-Cherenkov-type muon detector (MD) array have been successfully operated since 2014, at an altitude of 4,300 $\mathrm{m}$ in Tibet, China. we observed 24 gamma-ray events with energy greater than $100 \mathrm{TeV}$ against 5.5 background events, which corresponds to $5.6 \sigma$ statistical significance [四]. The highest energy of the detected gamma rays is estimated to be $450 \mathrm{TeV}$. This is the first detection of gamma rays beyond $100 \mathrm{TeV}$ from an astrophysical source, and a pioneering work opening a new higher energy window in the astronomy and astrophysics.

36th International Cosmic Ray Conference -ICRC2019-

July 24th - August 1st, 2019

Madison, WI, U.S.A.

${ }^{*}$ Speaker. 


\section{Observations of the Crab Nebula from TeV to sub-PeV}

The gamma rays beyond $100 \mathrm{TeV}$ has been previously undetected at the earth. The Crab Nebula is one of the most energetic astrophysical sources in the all sky, and its energy spectrum has been widely measured from radio to near $100 \mathrm{TeV}$ gamma rays. It is well known that the multiwavelength non-thermal energy spectrum is dominated by synchrotron radiation at energies lower than $1 \mathrm{GeV}$ and by the inverse-Compton scattering above $1 \mathrm{GeV}[[2, \mathrm{l}]$.

$\mathrm{TeV}$ gamma-rays from the Crab Nebula were first clearly detected by the Whipple collaboration using an imaging air Cherenkov telescope (IACT) in 1989 [四]. Since then, IACT has become the standard telescope for $\mathrm{TeV}$ gamma-ray observations by virtue of its excellent angular resolution and efficiency. The MAGIC experiment, equipped with the large reflector (17 $\mathrm{m}$ in diameter), has measured the lower energy part of the spectrum down to $77 \mathrm{GeV}$ [G]. In the energy above $10 \mathrm{TeV}$, The HEGRA experiment had obtained the energy spectrum up to $75 \mathrm{TeV}$ with an approximate single power-law [ []]. On the other hand, the H.E.S.S. experiment reported the cutoff energy $E_{\mathrm{c}}=\left(14.3 \pm 2.1_{\text {stat }}\right) \mathrm{TeV}$ in the energy spectrum of the Crab Nebula, assuming a power-law with an exponential cutoff $E^{-p} \exp \left(-E / E_{\mathrm{c}}\right)$, [U]. At the highest energies between $141 \mathrm{TeV}$ and $646 \mathrm{TeV}$, the CASA-MIA experiment have set flux upper limits to the gamma rays from the Crab Nebula by a large air-shower array with area of $230,000 \mathrm{~m}^{2}$ and the muon detector (MD) array, which consists of 1024 scintillation counters with an area of $2.5 \mathrm{~m}^{2}$ [ [8].

The Tibet AS $\gamma$ experiment achieved the first successful observation of the Crab Nebula at a multi-TeV region in 1999, using the Tibet-HD (high density) array with an area of $5,175 \mathrm{~m}^{2}$ [Q]. Subsequently, the Tibet-III array with an area of 22,050 $\mathrm{m}^{2}$ has been operating since 1999. Using the data collected by this array for 1318.9 live days, we measured the energy spectrum of gamma rays from the Crab Nebula from 1.7-40 TeV [山्U]. In 2007, the proto-type water Cherenkov MD with an area of $100 \mathrm{~m}^{2}$ was constructed under the Tibet AS array. We searched for gamma rays from the Crab Nebula above $100 \mathrm{TeV}$ using the background rejection technique by means of the counting the number of muons in the air shower. In spite of small MD compared with CASA-MIA experiment, The most stringent flux upper limit above $140 \mathrm{TeV}$ was obtained using the dataset collected for 438 live days[ए]].

In the begining of 2014 , the water-Cherenkov-type MD with the are of $3,400 \mathrm{~m}^{2}$ in total started operation. Based on the criterion of muon number measured in an air shower by the MD, we successfully suppress $99.9 \%$ of the cosmic-ray background events with energies $E>100 \mathrm{TeV}$. As a result, 24 gamma-like events with $E>100 \mathrm{TeV}$ were observed from the Crab Nebula against 5.5 cosmic-ray background events, corresponding to $5.6 \sigma$ statistical significance, with the Tibet AS array and the underground water-Cherenkov-type MD array (Tibet AS+MD array) [W]. This is the first detection of the highest energy gamma rays beyond $100 \mathrm{TeV}$ from an astrophysical source, and a pioneering work opening a new higher energy window in the astronomy and astrophysics. Recently, the HAWC also reported an indication of gamma rays above $100 \mathrm{TeV}$ at the significance $3.3 \sigma$ [ए2]. The flux around $100 \mathrm{TeV}$ measured by the Tibet AS+MD is consistent with the flux measured by the HAWC. In the near future, the detection of gamma rays above $100 \mathrm{TeV}$ is a key to solve the very-high-energy cosmic-ray origin which has been a mystery for more than hundred years since their discovery. 


\section{Integral Energy Spectrum in sub-PeV}

We calculated the integral fluxes for the Crab nebula to be $F(>100 \mathrm{TeV})=(3.29+1.06-$ $0.87) \times 10^{-15} \mathrm{~cm}^{-2} \mathrm{~s}^{-1}$ and $F(>250 \mathrm{TeV})=(5.72+5.72-3.48) \times 10^{-15} \mathrm{~cm}^{-2} \mathrm{~s}^{-1}$, respectively, to compare with the upper limits measured by past experiments in the sub-PeV energy region [四]. Figure $\square$ shows the integral energy spectrum observed by the Tibet AS+MD array and the previous upper limits at the $90 \%$ C.L. measured by the CASA-MIA experiment (black arrows with diamond marks) and Tibet AS with $100 \mathrm{~m}^{2}$ prototype MD (magenta arrows with plus marks). The measured integral fluxes by the Tibet AS+MD array are consistent with all the previous upper limits. At the energy above $600 \mathrm{TeV}$, the CASA-MIA upper limit partly constrains the $1 \sigma$ error region with the simple extrapolations of observed fluxes. The Tibet AS+MD will continuously observe the gamma rays from the Crab Nebula to extend the spectrum toward higher energy region up to $\mathrm{PeV}$.

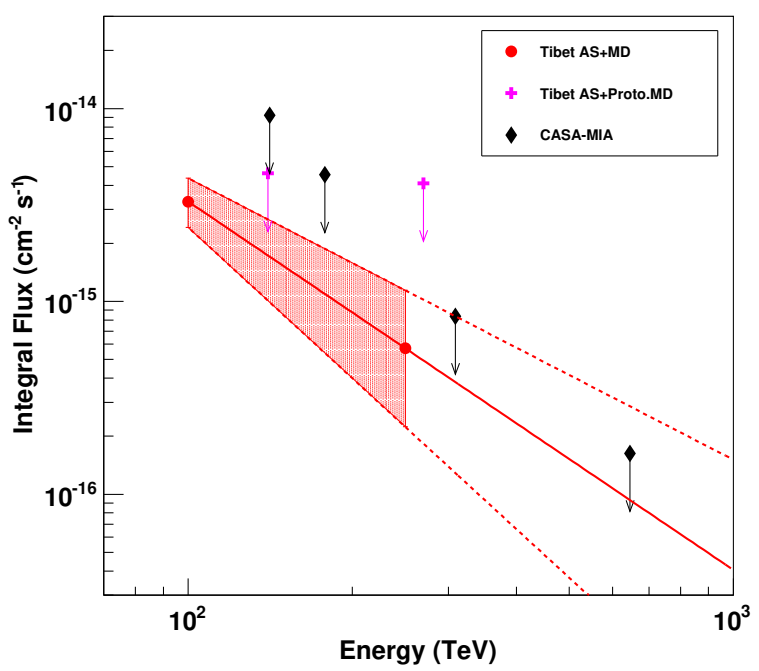

Figure 1: Integral energy spectrum in the sub-PeV region observed by the Tibet AS+MD array and the previous upper limits at the $90 \%$ C.L. measured by the CASA-MIA experiment (black arrows with diamond marks) and Tibet AS with $100 \mathrm{~m}^{2}$ prototype MD (magenta arrows with plus marks). The shaded area is $1 \sigma$ error region by the Tibet AS+MD array. The solid line shows simple extrapolation of observed fluxes by the Tibet AS+MD array, while the dashed lines show simple extrapolations of $1 \sigma$ errors.

\section{Acknowledgments}

The collaborative experiment of the Tibet Air Shower Arrays has been conducted under the auspices of the Ministry of Science and Technology of China and the Ministry of Foreign Affairs of Japan. This work was supported in part by a Grant-in-Aid for Scientific Research on Priority Areas from the Ministry of Education, Culture, Sports, Science, and Technology, by Grants-in-Aid for Science Research from the Japan Society for the Promotion of Science in Japan. This work is supported by the National Key R\&D Program of China (No. 2016YFE0125500). This work is supported by the Grants from the National Natural Science Foundation of China (No. 11533007, No. 11673041, and No. 11873065), and by the Key Laboratory of Particle Astrophysics, Institute 
of High Energy Physics, CAS. This work is supported by the joint research program of the Institute for Cosmic Ray Research (ICRR), the University of Tokyo.

\section{References}

[1] M. Amenomori, et al. First detection of photons with energy beyond $100 \mathrm{TeV}$ from an astrophysical source, Accepted for publication in PRL, in press (2019)

[2] O. C. De Jager, et al. Gamma-ray observations of the Crab Nebula: A study of the synchro-Compton spectrum, ApJ, 457, 253 (1996).

[3] A. M. Atoyan, and F. A. Aharonian, F. A On the fluxes of inverse Compton gamma-rays expected from the Crab Nebula, A\&A Suppl. Ser., 120, 453 (1996).

[4] T. C. Weekes, et al. Observation of TeV gamma rays from the Crab nebula using the atmospheric Cerenkov imaging technique, ApJ, 342, 379 (1989).

[5] F. Aharonian, et al. The Crab Nebula and pulsar between $500 \mathrm{GeV}$ and $80 \mathrm{TeV}$ : observations with the HEGRA stereoscopic air Cerenkov telescopes, ApJ, 614, 897 (2004).

[6] J. Albert, et al. VHE $\gamma$-ray observation of the Crab Nebula and its pulsar with the MAGIC telescope, ApJ, 674, 1037 (2008).

[7] F. Aharonian, et al. Observations of the Crab nebula with HESS, A\&A, 457, 899 (2006).

[8] A. Borione, et al. A search for ultra-high-energy gamma-Ray emission from the Crab Nebula and pulsar, ApJ, 481, 313 (1997).

[9] M . Amenomori, et al. Observation of Multi-TeV gamma rays from the Crab Nebula using the Tibet air shower array, ApJ, 525, L93 (1999).

[10] M. Amenomori, et al. Multi-TeV gamma-Ray observation from the Crab Nebula using the Tibet-III air shower array finely tuned by the cosmic-ray Moon's shadow, ApJ, 692, 61 (2009).

[11] M. Amenomori, et al. Search for Gamma Rays above $100 \mathrm{TeV}$ from the Crab Nebula with the Tibet Air Shower Array and $100 \mathrm{~m}^{2}$ Muon Detector, ApJ, 813, 98 (2015).

[12] A. U. Abeysekara, et al. Measurement of the Crab Nebula at the Highest Energies with HAWC, arXiv:1905.12518 (2019) 